\title{
A three dimensional model for the mechanical study of railway catenaries
}

\author{
J. Benet ${ }^{1}$, T. Rojo ${ }^{2}$, F. Cuartero ${ }^{2} \&$ E. Arias $^{2}$ \\ ${ }^{I}$ Department of Applied Mechanics, \\ University of Castilla-La Mancha, Spain \\ ${ }^{2}$ Albacete Research Institute of Informatics, \\ University of Castilla-La Mancha, Spain
}

\begin{abstract}
The increasing demands for rail units, requiring among other aspects optimal and accurate conditions to mount the catenary, needs a rigorous mechanical calculation where the development of the corresponding computer software is essential in order to solve the various problems in a satisfactory manner. Most of the work done at the moment are based on two-dimensional models, but that model is not quite realistic because the catenary is a structural system, consisting of wires arranged in a zigzag, considering different types of loading as the weight of the wires or the wind effect, acting such charges in different directions. The development of an advanced model in three dimensions can be of great help to achieve more realistic results. This paper is an introduction to the study of mechanical railway catenary based on a three-dimensional model, the method presented here allows different types of static problems to be solved, such as the calculation of the stiffness of the line and the calculation of the length of the droppers.
\end{abstract}

Keywords: railway catenary, static problems, three dimensional models, length of the droppers, effect of the wind.

\section{Introduction}

In order to obtain an adequate performance in the circulation of the railway units, the pantograph-catenary contact force should be maintained as uniform as possible, avoiding the lost of contact or take offs. This requires, among other aspects, precise conditions of assembly and a suitable configuration of the aerial 
line of contact or catenary. The requirements, every time increasing, for the railway units, in speed, reliability, etc., make it necessary to work on more complex and realistic models. The development of an advanced mathematical model that allows us to evaluate the mechanical behaviour of the system can be helpful in order to obtain optimal conditions of assembly.

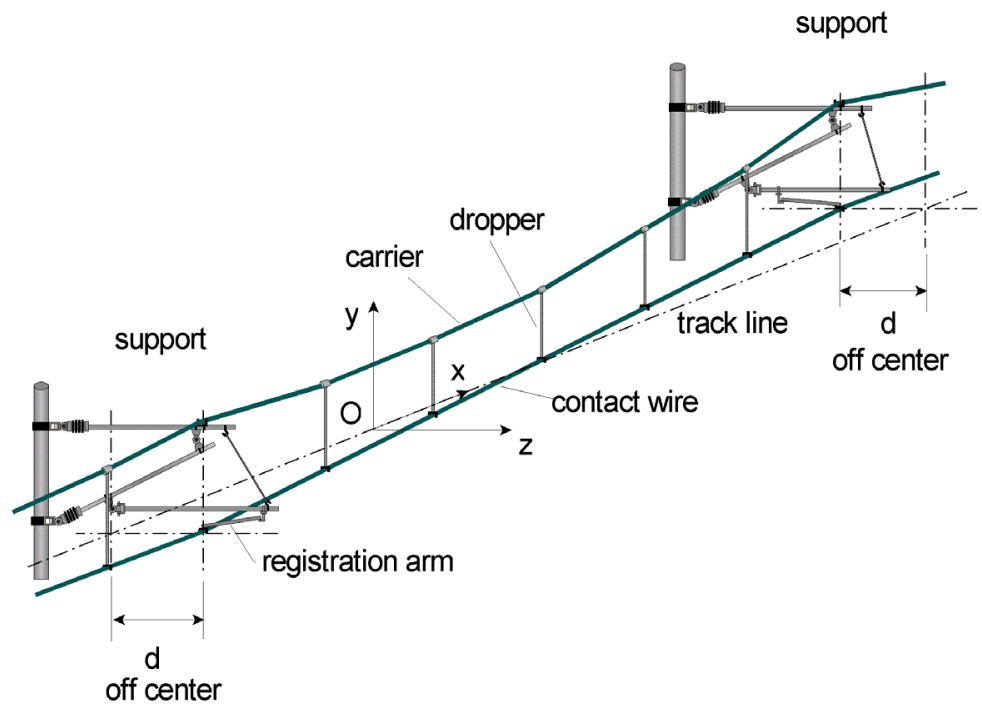

Figure 1: A span of catenary.

In the mechanical study of the aerial line of contact, different types of problems can be considered: calculation of the length of the droppers, to study the stiffness of the line, or to study the pantograph-catenary dynamic interaction. In most of the works realised until now, either in the study of problems of static type (see Galleotti and Toni [6] and Buffarini [7]), or of dynamic type (see Arnold and Simeon [1], and Benet et al [3]), the models are based on two dimensions. Nevertheless, this kind of model is not real, since the catenary is a structural system formed by cables, with lines distributed in zigzag, in the case of straight trajectories; or making a polygonal line, in the case of curved trajectories, with different types of load: weight of cables, effect of wind, acting in different directions, and so on.

Due to all these reasons, the traditional model of two dimensions becomes insufficient to solve in an adequate way the previous problems, being necessary the development of a more advanced model, considering three dimensions. The present work is an introduction to the mechanical study of railway catenary wires in three dimensions. A mathematical model based on the Finite Element Method (FEM) has been developed, which allows us to obtain the equations of static balance, from which different problems can be solved, such as the calculation of the stiffness of the line, and the length of the droppers. 


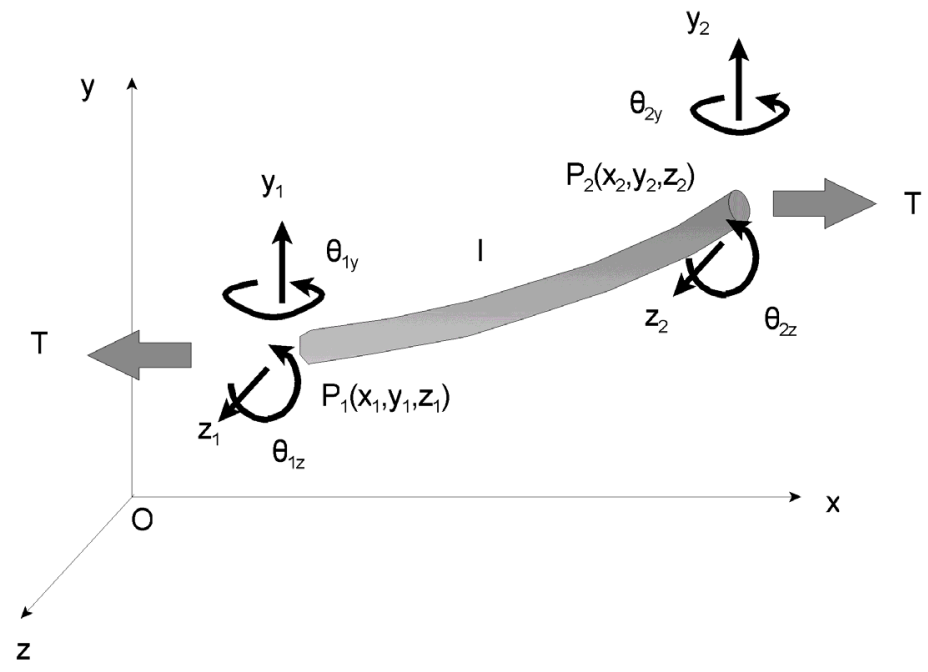

Figure 2: A pre-stressed beam.

\section{Model of the contact wire and carrier}

The aerial line of contact, or catenary, is modelled considering a series of spans, normally 15 or 20 , constituting each series an independent system. The spans usually present, in addition, a length of about $60 \mathrm{~m}$. In the European railways two types of assemblies can be basically considered: the normal catenary system, and the stitched catenary system.

In fig. 1 is showed a span of a normal catenary, in which three types of cables can be distinguished: the carrier, the droppers, and the contact wire. Both wires, the carrier and the contact wire, are tightened by pulleys and independent counterbalances, located at the ends of each series. The catenary is, then, a continuous system that can be modelled applying the techniques of FEM analysis, in agreement with is indicated by Bathe [2] and Cook et al [5].

With regard to carrier and contact wire, their model is obtained from the Euler-Bernouilli equation for a prestressed flexible beam under a vertical force of its own weight, and under the lateral effect of the wind. For the system of axes of the figure an element of the wire, represented in fig. 2, is considered like a prestressed beam, presenting four coordinates generalized by node: the displacement and the angle according to the y-axis, and the z-axis. The equation of balance for a cable element can be represented as a system of eight linear equations, which can be expressed of generic form as:

$$
f_{c}=k_{c} q_{c}-r_{c}=\left(k_{c 1}+k_{c 2}\right) q_{c}-r_{c} .
$$

Where $\mathrm{f}_{\mathrm{c}}$ is the resulting vector of the forces and external moments applying at the nodes, $\mathrm{k}_{\mathrm{c}}$ represents the stiffness matrix of the element, which can be 
spitted in two matrices, the first one, $\mathrm{k}_{\mathrm{c} 1}$, is the stiffness matrix corresponding to the elastic potential of flexion, and being $\mathrm{k}_{\mathrm{c} 2}$ the stiffness matrix corresponding to the pre-stressed potential, and $r_{c}$ is the vector corresponding to the vertical load (weight) and the lateral load (wind), being the components from the eqn. (1) for an element of length 1 :

$$
r_{c}=\left(\begin{array}{c}
-p_{y} l / 2 \\
-p_{y} l^{2} / 12 \\
-p_{y} l / 2 \\
p_{y} l^{2} / 12 \\
p_{z} l / 2 \\
-p_{z} l^{2} / 12 \\
p_{z} l / 2 \\
p_{z} l^{2} / 12
\end{array}\right), \quad k_{c 1}=\frac{E . I}{l^{3}}\left(\begin{array}{cccccccc}
12 & 6 l & -12 & 6 l & 0 & 0 & 0 & 0 \\
6 l & 4 l^{2} & -6 l & 2 l^{2} & 0 & 0 & 0 & 0 \\
-12 & -6 l & 12 & -6 l & 0 & 0 & 0 & 0 \\
6 l & 2 l^{2} & -6 l & 4 l^{2} & 0 & 0 & 0 & 0 \\
0 & 0 & 0 & 0 & 12 & -6 l & -12 & -6 l \\
0 & 0 & 0 & 0 & -6 l & 4 l^{2} & 6 l & 2 l^{2} \\
0 & 0 & 0 & 0 & -12 & 6 l & 12 & 6 l \\
0 & 0 & 0 & 0 & -6 l & 2 l^{2} & 6 l & 4 l^{2}
\end{array}\right) .
$$

For the component of the stiffness matrix $\mathrm{k}_{\mathrm{c} 2}$, corresponding to the prestressed potential of the wire, and for the generalized coordinates vector:

$$
q_{c}=\left(\begin{array}{c}
y_{1} \\
\theta_{1 y} \\
y_{2} \\
\theta_{2 y} \\
z_{1} \\
\theta_{1 z} \\
z_{2} \\
\theta_{2 z}
\end{array}\right), \quad k_{c 2}=\frac{T}{30 l}\left(\begin{array}{cccccccc}
36 & 3 l & -36 & 3 l & 0 & 0 & 0 & 0 \\
3 l & 4 l^{2} & -3 l & -l^{2} & 0 & 0 & 0 & 0 \\
-36 & -3 l & 36 & -3 l & 0 & 0 & 0 & 0 \\
3 l & -l^{2} & -3 l & 4 l^{2} & 0 & 0 & 0 & 0 \\
0 & 0 & 0 & 0 & 36 & -3 l & -36 & -3 l \\
0 & 0 & 0 & 0 & -3 l & 4 l^{2} & 3 l & -l^{2} \\
0 & 0 & 0 & 0 & -36 & 3 l & 36 & 3 l \\
0 & 0 & 0 & 0 & -3 l & -l^{2} & 3 l & 4 l^{2}
\end{array}\right) .
$$

Being $p_{y}$ the vertical weight of the wire by length unit, $p_{z}$ the load of the wind by length unit (positive o negative), $T$ the mechanical tension of the wire, I the moment of diametrical inertia and $\mathrm{E}$ the elastic module of the material.

\section{Model of droppers}

The droppers behave like elastic traction bars, with an initial length $1_{0}$, and stretched a small quantity $\Delta \mathrm{l}$, reaching a final length of 1 , according with the outline presented in fig. 3. In this case, each generalized node has two coordinates, corresponding to displacements according to the y-axis and, the zaxis, not experiencing any variation depending on the x-axis. The equations of static equilibrium will be:

$$
\left.\begin{array}{l}
f_{1 y}=\left(E A / l_{0}\right)\left(y_{1}-y_{2}-l_{0} \cos \varphi\right)+p_{y} l_{0} / 2, \\
f_{2 y}=-\left(E A / l_{0}\right)\left(y_{1}-y_{2}-l_{0} \cos \varphi\right)+p_{y} l_{0} / 2, \\
f_{1 z}=\left(E A / l_{0}\right)\left(z_{1}-z_{2}-l_{0} \sin \varphi\right)-p_{z} l_{0} / 2, \\
f_{2 z}=-\left(E A / l_{0}\right)\left(z_{1}-z_{2}-l_{0} \cos \varphi\right)-p_{z} l_{0} / 2 .
\end{array}\right\}
$$



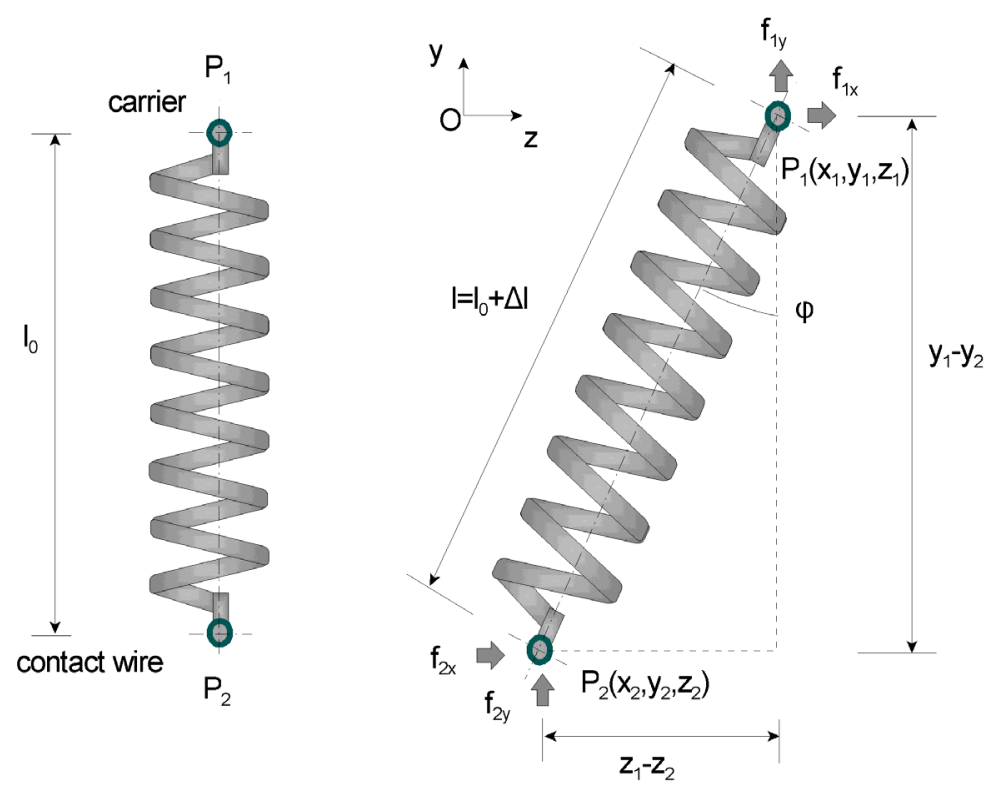

Figure 3: $\quad$ Model of a dropper.

These equations can be written in matrix form, similar to eqn. (1), where:

$$
q_{d}=\left(\begin{array}{l}
y_{1} \\
y_{2} \\
z_{1} \\
z_{2}
\end{array}\right), k_{d}=\frac{E A}{l_{0}} \cdot\left(\begin{array}{cccc}
1 & -1 & 0 & 0 \\
-1 & 1 & 0 & 0 \\
0 & 0 & 1 & -1 \\
0 & 0 & -1 & 1
\end{array}\right), r_{d}=\left(\begin{array}{c}
E A \cos \varphi-p_{y} l_{0} / 2 \\
-E A \cos \varphi-p_{y} l_{0} / 2 \\
E A \sin \varphi+p_{z} l_{0} / 2 \\
-E A \sin \varphi+p_{z} l_{0} / 2
\end{array}\right) .
$$

Where $\mathrm{E}$ is the elastic module of the material, A the area of the section, $\mathrm{p}_{\mathrm{y}}$, the downwards force by unit of length due to the weight of the dropper, $p_{z}$ is the lateral force by unit of length due to the wind (positive or negative) and $\varphi$ the inclination angle of the dropper with respect to the vertical. $\mathrm{P}_{1}\left(\mathrm{x}_{1}, \mathrm{y}_{1}, \mathrm{z}_{1}\right)$ and $\mathrm{P}_{2}\left(\mathrm{x}_{2}, \mathrm{y}_{2}, \mathrm{z}_{2}\right)$ represent the extreme nodes of the dropper, according with fig. 3 , and also satisfying:

$$
\sin \varphi=\frac{z_{1}-z_{2}}{\sqrt{\left(y_{1}-y_{2}\right)^{2}+\left(z_{1}-z_{2}\right)^{2}}}, \quad \cos \varphi=\frac{y_{1}-y_{2}}{\sqrt{\left(y_{1}-y_{2}\right)^{2}+\left(z_{1}-z_{2}\right)^{2}}} .
$$

Furthermore, the droppers work always by traction, so that their effect on the static or dynamic equations only is considered when the actual length, taken as the extreme distance between nodes, is greater than or equal to the initial length $1_{0}$, determined in a previous static calculation, that is:

$$
\sqrt{\left(z_{1}-z_{2}\right)^{2}+\left(y_{1}-y_{2}\right)^{2}} \geq l_{0} \text {. }
$$




\section{Equations of static equilibrium}

According with all the explained above, the equation of the static equilibrium of the catenary can be expressed by combining the equilibrium equations of each element, corresponding to the decomposition by the FEM, and equalling to zero the resulting composition of the forces:

$$
F=K(q) q-R(q)=0 .
$$

Where the elements in the equations are:

$$
F=\sum f, \quad K(q)=\sum k, \quad R(q)=\sum r(q) .
$$

The eqn. (8), corresponding to the static equilibrium condition of the catenary is actually a nonlinear equations system, where $\mathrm{K}(\mathrm{q})$ represents the stiffness matrix of the catenary. This matrix, obtained as a sum of the stiffness matrices of the elements, is not a constant matrix, because for a given state of load, it has to be included in this addition the corresponding stiffness matrices of the droppers working on traction, which is impossible to know beforehand. The matrix $K(q)$ is a sparse matrix, being advisable the use of any specific software package in accordance with Duff et al [4], to solve eqn. (8), in order to obtain a greater computational efficiency.

The independent term $\mathrm{R}(\mathrm{q})$ is also variable, because the independent terms of the corresponding droppers, depend on the coordinates of the extreme nodes of the droppers, according with eqns. (5) and (6). Moreover, as it occurs with the stiffness matrix, it only has to be included the independent terms of the droppers working on traction.

Also, it is possible to determine the position of equilibrium in the catenary, for a given state of load, by solving eqn. (8) of static equilibrium, using an iterative method. An algorithm defining the stiffness matrix and the independent term for each step has been developed. We have supposed a state of loads due to the lateral action of the wind, the weight of the elements, and an upward force on time, as it may be the thrust of the pantograph, and also taking into account the disconnection of the droppers. According with this, the algorithm to calculate the position of equilibrium is:

1. The stiffness matrix of the catenary $K(q)$ is configured, with the initial assumption that all the droppers are working on traction.

2. It is further assumed initially that the ends of the droppers are on the same vertical line, this means that $\varphi=0$ in eqn. (5), setting the independent term, represented by $\mathrm{R}_{0}(\mathrm{q})$.

3. For the aforementioned assumptions, it is solved the equation in the form of the linear system:

$$
K(q) \cdot q_{i+1}-R_{i}(q)=0 .
$$

where the subscript $i$ represents the iteration counter, initially $i=0$.

4. It is compared the solution of eqn. (9), $\mathrm{q}_{i+1}$, corresponding to the iteration $\mathrm{i}$ +1 , with the solution obtained in the previous iteration, represented by $\mathrm{q}_{\mathrm{i}}$. If the difference is below a certain error value, the last solution is considered the 
correct solution of (9) for the supposed number of connected droppers, on the contrary, it is set up the independent term $R_{i+1}(q)$ with the obtained value of $q_{i+1}$, repeating the cycle until the error is below a certain value.

5. The obtained solution of eqn. (9) is valid for the assumed number of connected droppers (originally it was considered that all the droppers were working on traction), but this assumption can be changed for the new solution obtained. For this, the droppers working on traction from the last position of balance calculated, have to be determined, verifying that the actual length is greater than or equal to the initial length, according to eqn. (7). This condition should be checked for all droppers supposed working on traction before, and the stiffness matrix $\mathrm{K}(\mathrm{q})$ and the independent term $\mathrm{R}(\mathrm{q})$ must be reconfigured for the new condition, coming back to point 3 and repeating the process again. In the case that the condition (7) is fulfilled for all the droppers supposed initially working on traction, the last solution obtained from (9) is the final solution for the position of equilibrium.

\section{Calculation of the length of the droppers}

With the calculation of the equilibrium positions of the wires, another important static issue related to the assembly of the line, consists in determining the length of the droppers, so that the contact wire acquires a particular configuration previously imposed. Although there are different ways to solve this problem, a possible method is to use eqn. (8), but making some modifications. Thus, in eqn. (5), corresponding to the terms of the equilibrium equation for the droppers, the coordinate $y_{2}$ of the node corresponding to the lower position of the dropper is now considered as a datum that corresponds to the position imposed on the contact wire: either parallel to the plane of the ground, either parabolic with a separation in the centre (pre-sag), etc. while the initial length $l_{0}$ becomes a variable, and the rest of the positions of the nodes of the wires also remain variable; on the other hand, the stiffness of the dropper which, in principle, has an unknown value $\mathrm{EA} / \mathrm{l}_{0}$, due that we do not know its original length $1_{0}$, should be replaced by a high-value $\left(10^{6} \mathrm{~N} / \mathrm{m}\right.$ or more $)$, represented by $\mathrm{k}$, then, the terms of eqn. (4) for the equilibrium of the droppers are:

$$
q_{d}=\left(\begin{array}{c}
y_{1} \\
l_{0} \\
z_{1} \\
z_{2}
\end{array}\right) \quad k_{d}=\left(\begin{array}{cccc}
k & -k \cos \varphi+p_{y} / 2 & 0 & 0 \\
-k & k \cos \varphi+p_{y} / 2 & 0 & 0 \\
0 & -k \sin \varphi & k & -k \\
0 & k \sin \varphi & -k & k
\end{array}\right), \quad r_{d}=\left(\begin{array}{c}
k \cdot y_{2} \\
-k \cdot y_{2} \\
0 \\
0
\end{array}\right) .
$$

Equations (2) and (3) relate the elements of prestressed beam type of the contact wire, it also should be changed for the coordinate $y_{1}$ or $y_{2}$ in the case in that the node element is jointed with a dropper, passing this coordinates to be a datum. Thus, it is possible to obtain the equilibrium equation in the form of a nonlinear equations system, similar to eqn. (8), which can be solved through an iterative method to determine the length of the droppers $1_{0}$; in this case it is assumed by hypothesis that all the droppers are connected and are working by traction, with absence of lateral effect of the wind. After determining the length 

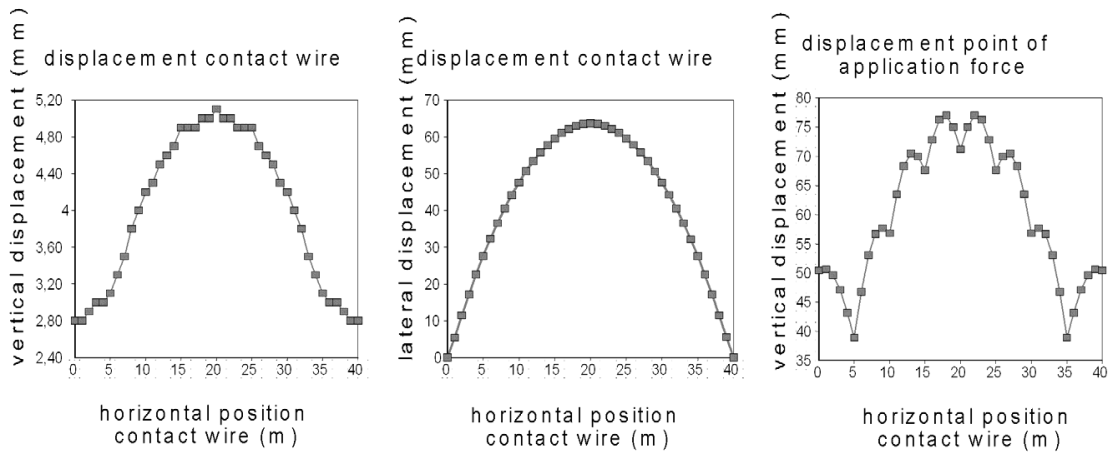

Figure 4: Offset of contact wire at the central span.

of the droppers, the traction force and the weight of the droppers can be calculated also.

\section{Applications}

The theoretical procedures explained before, allow us to assemble the stiffness matrix for a series of catenary spans and to determine the equilibrium configuration of the system, assuming different states of forces. A sequence of three catenary spans has been supposed, each one has $40 \mathrm{~m}$ of length, arranged in a zigzag, an off-centre of $0.2 \mathrm{~m}$, with copper wires of $12 \mathrm{~mm}$ diameter in the carrier and contact wire; a tension of $10000 \mathrm{~N}$ in both extremes of the carrier and contact wire, and seven droppers in the span, distributed in an equidistant way has been considered also; it has been assumed that the contact wire is horizontal and parallel to the plane of the ground.

With this characteristics, first, the length of the droppers has been calculated, by solving the eqn. (8) of static equilibrium, using eqn. (10), by the method proposed in the preceding section, obtained for the three spans, the lengths, forces and weights of the droppers given by table 1:

Table 1: $\quad$ Features of the droppers.

\begin{tabular}{|c|c|c|c|}
\hline $\begin{array}{c}\text { Dropper } \\
\text { number }\end{array}$ & Length $(\mathrm{mm})$ & Load force $(\mathrm{N})$ & $\begin{array}{c}\text { Weight force } \\
(\mathrm{N})\end{array}$ \\
\hline 1 & 1021.39 & 51.227 & 2.22 \\
\hline 2 & 893.95 & 50.94 & 1.94 \\
\hline 3 & 817.53 & 50.78 & 1.85 \\
\hline 4 & 792.09 & 50.724 & 1.73 \\
\hline 5 & 817.53 & 50.78 & 1.85 \\
\hline 6 & 893.95 & 50.94 & 1.94 \\
\hline 7 & 1021.39 & 51.227 & 2.22 \\
\hline
\end{tabular}


Then, the stiffness matrix of the system has been configured for the obtained length and weight of the droppers, and the equilibrium positions of the wires have been calculated, according to eqn. (8) for different states of forces. First, we calculate the equilibrium positions when the forces only were due to the weight of the wires. After that, we calculate again the position of equilibrium assuming wind acting in a positive direction in the z-axis of $80 \mathrm{~km} / \mathrm{h}$, causing a lateral force of $3.2 \mathrm{~N} / \mathrm{m}$ on the wires. The effect of the wind has produced a vertical and lateral displacement on the wires, which has been compared with the initial position without wind, having represented such a shift for the contact wire of the central span, corresponding to the first two graphs in fig. 4. It can be further noted that the wind does a considerable effect on the equilibrium configuration of the catenary, producing significant displacements, both vertical and horizontal, which can affect later in the pantograph-catenary interaction.

Finally, it has been assumed, in addition to the wind, an upwards force, as it may be the one produced by the pressure of the pantograph, of $120 \mathrm{~N}$, acting at different points along the central span, having represented in the third graphic the vertical displacement of the point of application of the force with respect to the starting position of the catenary without wind effect. This third chart is known as stiffness span curve, and we can see that the vertical displacement of the point of application of the force is much higher in the centre of the span than in the extreme. In this case, we can also observe that the effect of the wind does not have much influence on the shape of the stiffness curve.

\section{Conclusions}

In this paper, we have developed a model to make an advanced mechanical study of railway catenary in three dimensions. Most of the studies realized up to now, are based on two-dimensional models; but that model is not real, because the catenary is a structural system formed by cables with different configurations, depending on whether it is straight or curved paths, and different types of forces, as the weight of the wires or the side-effect wind. The presented model takes into account all these details, allowing the knowledge of the behaviour of the system in a completely realistic way.

In order to develop the model, the Finite Element Method has been applied, in which carrier and contact wires are treated as pre-stressed beams and droppers as stretched bars. The static equilibrium equations of the system have been established, obtaining a nonlinear equations system that can be solved by using an iterative method. It is also possible, with these equations, to calculate the length of the droppers for several configurations of the contact wire. As application, we have studied the equilibrium configuration of the line with different cases of forces, assuming the action side of the wind. The model presented can also be implemented in a computer tool, suitable for the study of the pantograph-catenary dynamic interaction, allowing us to obtain very realistic simulations. 


\section{References}

[1] Arnold, M. \& Simeon, B., Pantograph-Catenary Dynamics: A Benchmark Problem and its Numerical Solution, Applied Numerical Mathematics, 34(4), pp. 345-362, 2000.

[2] Bathe, K. J., Finite Element Procedures, Prentice Hall, 1996.

[3] Benet, J., Alberto, A., Arias, E. \& Rojo, T., A Mathematical Model of the Pantograph-Catenary Dynamic Interaction with Several Contact Wires, IAENG International Journal of Applied Mathematics, 37(2), pp. 136-144, December, 2007.

[4] Duff, I. S., Erisman, A. M. \& Reid, J. K., Direct Methods for Sparse Matrices, Claredon, Oxford, 1986.

[5] Cook, D. C., Malkus, D. S. \& Plesha, M. E., Concepts and Applications of Finite Element Analysis, John Wiley and Sons, 1989.

[6] Galleotti, G. \& Toni P., Overhead Contact line Elasticity Optimization for Railway High Speed Running, Computers and Structures, 65(6), pp. 975983, December, 1997.

[7] Buffarini, G., Calcolo dell'assetto e dell'elasticità statica, tramite modello elettrico, per catenarie con (o senza) disposizione a Y, Ingegneria Ferroviaria, Roma, 1987. 